\title{
How Flat Space Cosmology Models Dark Energy
}

\author{
Eugene Terry Tatum ${ }^{1}$, U. V. S. Seshavatharam ${ }^{2}$ \\ ${ }^{1}$ Independent Researcher, Bowling Green, KY, USA \\ ${ }^{2}$ Honorary Faculty, I-SERVE, S. No-42, Hitech City, Hyderabad, India \\ Email:ett@twc.com, seshavatharam.uvs@gmail.com
}

How to cite this paper: Tatum, E.T. and Seshavatharam, U.V.S. (2020) How Flat Space Cosmology Models Dark Energy. Journal of Modern Physics, 11, 1493-1501. https://doi.org/10.4236/jmp.2020.1110091

Received: September 3, 2020

Accepted: October 10, 2020

Published: October 13, 2020

Copyright $\odot 2020$ by author(s) and Scientific Research Publishing Inc. This work is licensed under the Creative Commons Attribution International License (CC BY 4.0).

http://creativecommons.org/licenses/by/4.0/

\section{(c) (i) Open Access}

\begin{abstract}
Equations of Flat Space Cosmology (FSC) are utilized to characterize the model's scalar temporal behavior of dark energy. A table relating cosmic age, cosmological redshift, and the temporal FSC Hubble parameter value is created. The resulting graph of the log of the Hubble parameter as a function of cosmological (or galactic) redshift has a particularly interesting sinuous shape. This graph greatly resembles what $\Lambda \mathrm{CDM}$ proponents have been expecting for a scalar temporal behavior of dark energy. And yet, the FSC $R_{h}=$ $c t$ model expansion, by definition, neither decelerates nor accelerates. It may well be that apparent early cosmic deceleration and late cosmic acceleration both ultimately prove to be illusions produced by a constant-velocity, linearly-expanding, FSC universe. Furthermore, as discussed herein, the FSC model would appear to strongly support Freedman et al. in the current Hubble tension debate, if approximately 14 Gyrs can be assumed to be the current cosmic age.
\end{abstract}

\section{Keywords}

Flat Space Cosmology, Dark Energy, Hubble Parameter, Galactic Redshift, $R_{h}=c t$ Model

\section{Introduction and Background}

We are currently in a "golden age" of astronomy and cosmology. Astrophysical observations in the coming decade are expected to bring much greater resolution concerning the behavior and fundamental nature of dark matter and dark energy. These are two of the remaining great mysteries of the universe.

With respect to the behavior of dark energy, the expansion history of our universe, going back to the earliest galaxies, should come into greater focus. If all goes well with these observations, we should be able to fill in many details with respect to the velocities of galactic separation going all the way back to the first 
few hundred million years of cosmic expansion. We should then have a remarkably accurate "moving picture" computer simulation of the history of that portion of the universe we can now observe.

When astrophysicists concern themselves with the velocities of galactic separation on scales greater than those of the local clusters held together by gravity and dark matter, they are studying the Hubble parameter and its tight correlation with cosmological redshift. When the Hubble parameter is characterized as a "snapshot" of the universe at a particular point in cosmic time (at the present time, for instance), it can be referred to as the Hubble constant. On a global scale, making use of cosmic microwave background (CMB) observations, the 2018 Planck Collaboration has arrived at a current Hubble constant $H_{0}$ value of $67.36+/-0.54 \mathrm{~km} \cdot \mathrm{s}^{-1} \cdot \mathrm{Mpc}^{-1}[1]$.

The ongoing temporal (i.e., "moving picture") studies of the universe are expected to show that, over the great span of cosmic time, the Hubble parameter is, in fact, scalar in some way. The first evidence of this became apparent in 1998, with studies of Type Ia supernovae [2], which revealed the presence of dark energy. Thus, it became apparent that there is an unseen energy, presumably within the cosmic vacuum, which prevents gravitational deceleration of the expanding universe. We now know that universal expansion, at present, is either occurring at constant velocity (as treated by $R_{h}=c t$ cosmological models) or very slightly accelerating (as claimed by $\Lambda \mathrm{CDM}$ concordance model cosmologists). Both types of cosmological models are still viable at the present time [3]-[8]. Observations in the coming decade may well identify which model is superior.

Flat Space Cosmology (FSC) is perhaps the most successful $R_{h}=c t$ model to date [9]. It predicts a current Hubble parameter $H_{0}$ value of $66.893 \mathrm{~km} \cdot \mathrm{s}^{-1} \cdot \mathrm{Mpc}^{-1}$, fitting with the 2018 Planck Collaboration consensus. It also predicts the COBE CMB $d T / T$ anisotropy ratio of $0.66 \times 10^{-5}$. A book chapter summary of FSC is now freely available online [10]. In contrast to $\Lambda \mathrm{CDM}$ cosmology (which incorporates observations ad hoc but makes relatively few falsifiable predictions), the FSC equations provide for very specific predictions, which can falsify the model if proven wrong. Remarkably, to date, the FSC model has not been falsified.

The purpose of the current report is to show how FSC models the temporal dark energy expansion of the universe. We show in great detail the scalar nature of the FSC Hubble parameter, so that it can be compared to the observations to be made in the coming decade.

\section{Methods}

Previously-published equations of FSC, relating cosmological (or galactic) redshift $z$, temporal cosmic temperature $T_{t}$ temporal cosmic radius $R_{t}$ the associated temporal Hubble parameter $H_{t}$ the currently-observed Hubble parameter $H_{o}$, the currently-observed cosmic temperature $T_{o}$, and cosmic age $t$, are brought together in the Results section in order to derive the parameter values given in Table 1 and Figure 1. 


\section{Results}

The following two FSC equations are useful for deriving the model relationships between a given cosmological (or galactic) redshift $z$ and the associated temporal Hubble parameter $H_{t}$ :

$$
z \cong\left(\frac{T_{t}^{2}}{T_{o}^{2}}-1\right)^{1 / 2}
$$

and

$$
T_{t}^{2} R_{t} \cong 1.027246639815497 \times 10^{27} \mathrm{~K}^{2} \cdot \mathrm{m}
$$

The first equation relates the redshift to the temporal cosmic temperature $T_{t}$ and the currently-observed cosmic temperature $T_{o}[11]$. The second equation relates the temporal cosmic temperature $T_{t}$ to the temporal cosmic radius $R_{t}$ [12].

Recalling the FSC Hubble parameter definition $\left(H_{t}=c / R_{t}\right)$, rearrangement and substitution gives:

$$
T_{0}^{2}\left(z^{2}+1\right) \cong H_{t}\left[\frac{1.027246639815497 \times 10^{27} \mathrm{~K}^{2} \cdot \mathrm{m}}{c}\right]
$$

To convert the $H_{t}$ term from reciprocal seconds $\left(\mathrm{s}^{-1}\right)$ to the conventional Hubble parameter units of $\mathrm{km} \cdot \mathrm{s}^{-1} \cdot \mathrm{Mpc}^{-1}$, the left-hand term is multiplied by $3.08567758 \times 10^{19} \mathrm{~km} \cdot \mathrm{Mpc}^{-1}$ :

$$
\begin{aligned}
& T_{0}^{2}\left(z^{2}+1\right)\left(3.08567758 \times 10^{19} \mathrm{~km} \cdot \mathrm{Mpc}^{-1}\right) \\
& \cong H_{t}\left[\frac{1.027246639815497 \times 10^{27} \mathrm{~K}^{2} \cdot \mathrm{m}}{c}\right]
\end{aligned}
$$

Rearrangement of terms gives:

$$
\left(z^{2}+1\right) \cong H_{t}\left[\frac{1.027246639815497 \times 10^{27} \mathrm{~K}^{2} \cdot \mathrm{m}}{T_{0}^{2} c\left(3.08567758 \times 10^{19} \mathrm{~km} \cdot \mathrm{Mpc}^{-1}\right)}\right]
$$

Using $T_{0}=2.72548 \mathrm{~K}$, this simplifies to:

$$
H_{t} \cong \frac{\left(z^{2}+1\right)}{0.014949183831548}
$$

The final useful equation relates cosmic time $t$ (in Gyrs after the Planck epoch) to the current Hubble parameter $H_{0}$ value of $66.893 \mathrm{~km} \cdot \mathrm{s}^{-1} \cdot \mathrm{Mpc}^{-1}$, the temporal Hubble parameter $H_{t}$ value, and the current FSC cosmic age of 14.617 Gyrs:

$$
H_{t} \cong H_{0}\left(\frac{14.617}{t}\right)
$$

Equations (5), (6) and (7) can then be used to create Table 1 and Figure 1. The last two $z$ values given in Table 1 are two of the highest galactic redshifts observed to date. 
Table 1. Cosmic age, Redshift $z$, Hubble parameter, $\log _{10}$ Hubble parameter.

\begin{tabular}{|c|c|c|c|}
\hline Cosmic Age (Gyrs) & Redshift $z$ & $H_{t}\left(\mathrm{~km} \cdot \mathrm{s}^{-1} \cdot \mathrm{Mpc}^{-1}\right)$ & $\log _{10}\left(H_{t}\right)$ \\
\hline 14.617 & 0.00 & 66.893 & 1.83 \\
\hline 14 & 0.21 & 69.84 & 1.84 \\
\hline 13.8 & 0.24 & 70.85 & 1.85 \\
\hline 13 & 0.35 & 75.21 & 1.88 \\
\hline 12 & 0.47 & 81.48 & 1.91 \\
\hline 11 & 0.57 & 88.89 & 1.95 \\
\hline 10 & 0.68 & 97.78 & 1.99 \\
\hline 9 & 0.79 & 108.64 & 2.04 \\
\hline 8 & 0.91 & 122.22 & 2.09 \\
\hline 7 & 1.04 & 139.68 & 2.15 \\
\hline 6 & 1.20 & 162.96 & 2.21 \\
\hline 5 & 1.39 & 195.55 & 2.29 \\
\hline 4 & 1.63 & 244.44 & 2.39 \\
\hline 3 & 1.97 & 325.92 & 2.51 \\
\hline 2 & 2.51 & 488.89 & 2.69 \\
\hline 1 & 3.69 & 977.77 & 2.99 \\
\hline 0.5 & 5.31 & 1955.55 & 3.29 \\
\hline 0.25 & 7.58 & 3911.1 & 3.59 \\
\hline 0.174 & 9.11 & 5618.51 & 3.75 \\
\hline 0.1179 & 11.09 & 8293.97 & 3.92 \\
\hline
\end{tabular}

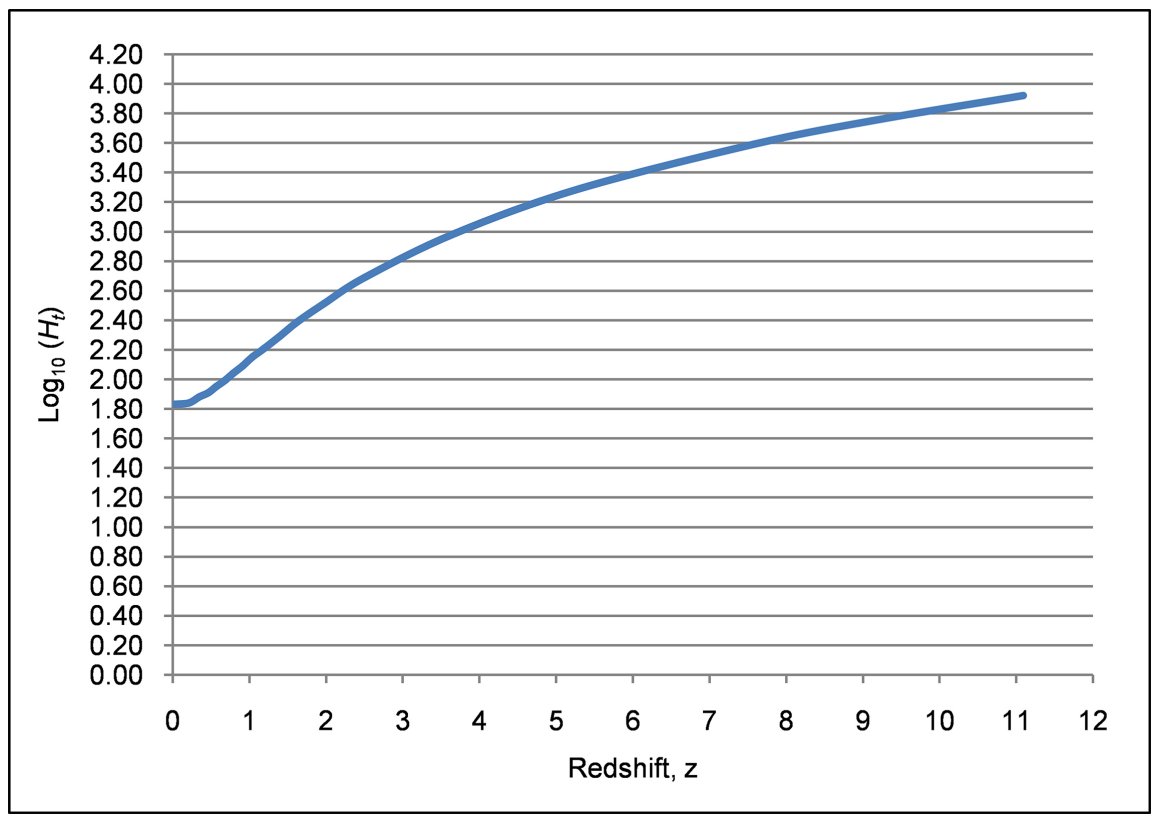

Figure 1. $\log _{10}\left(H_{t}\right)$ as a function of cosmological (or galactic) redshift $z$. 
Notice the sinuous appearance of this graph. Its overall shape greatly resembles what cosmologists have been expecting for a scalar temporal behavior of dark energy!

\section{Discussion}

Proponents of the $\Lambda \mathrm{CDM}$ concordance model of cosmology, and $R_{h}=c t$ model cosmologists, are currently in a pitched battle to establish which model is more accurate with respect to observations and predictions. As documented in recent publications [13] [14], FSC is a realistic linear light-speed cosmic expansion model which can also be considered a modified Milne "empty universe" model. Following a sign convention which treats gravitationally-attracting matter energy density as positive and "repulsive gravity" vacuum energy density as negative, the FSC net global energy density is perpetually zero. Thus, the FSC cosmic model follows the "empty" line exactly between deceleration and acceleration in this Figure 2 open source graph [15] from the Supernova Cosmology Project. One can readily see that the observational error bars allow for BOTH models [i.e., the blue line of $\Lambda C D M$ accelerating expansion, as well as the "empty" pink line corresponding to constant velocity expansion of the FSC $R_{h}=c t$ model].

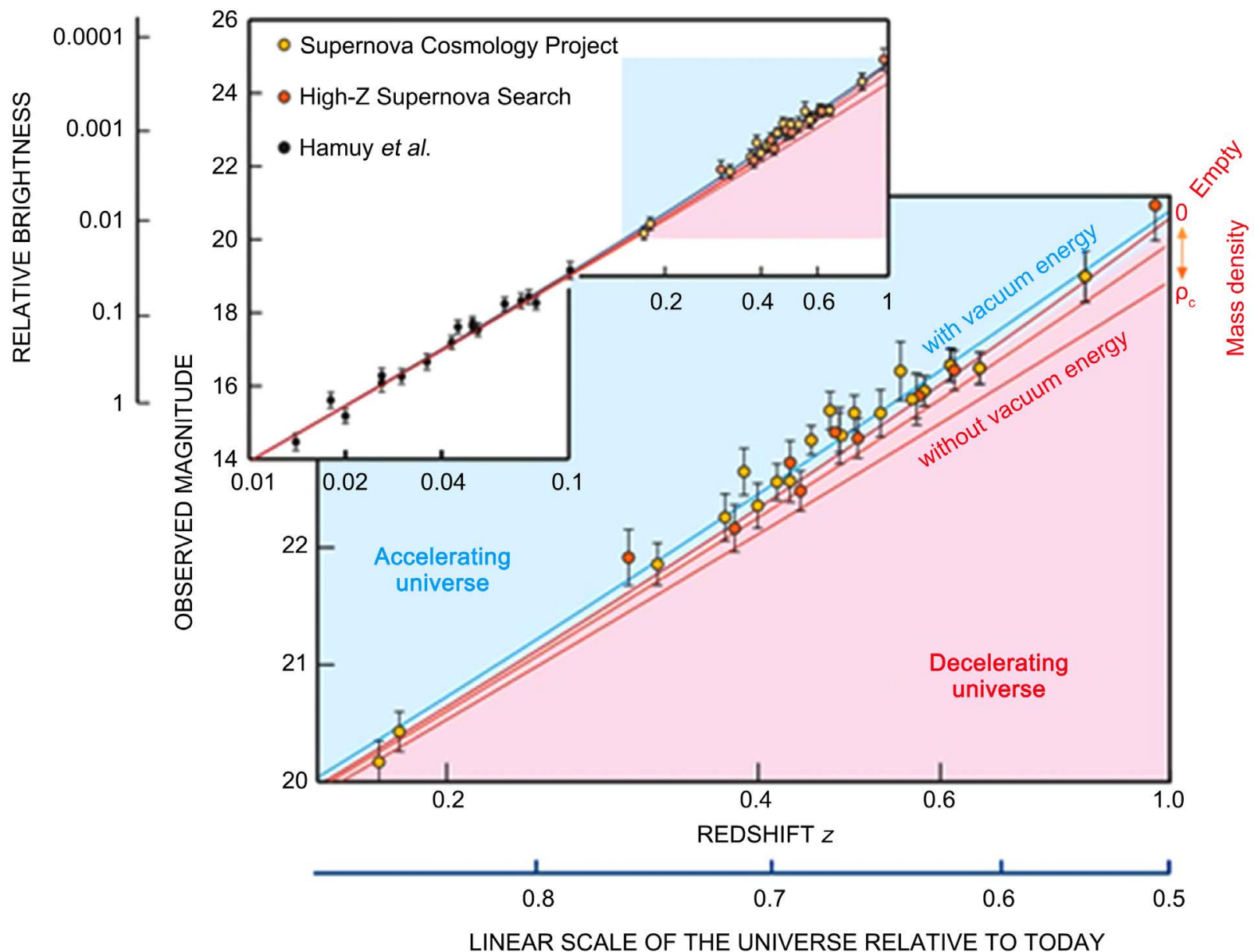

Figure 2. Observed magnitudes of type Ia supernovae vs redshift $z$. 
Notice also that this graph correlates a redshift $z$ value of 1.0 with a cosmic scale of 0.5 times the current scale. This is true for FSC as well as $\Lambda \mathrm{CDM}$, although the two models differ slightly with respect to the current cosmic age.

In $\Lambda$ CDM cosmology, the post-inflationary cosmological vacuum energy density is assumed to be a constant. This is not an absolute requirement of general relativity, so long as the vacuum energy density is scalar according to $\Lambda=3 H_{t}^{2} / c^{2}$. In the FSC quintessence model, this scalar relationship holds true and is equivalent to $\Lambda=3 / R_{t}^{2} \quad$ [16]. In FSC, the vacuum energy density declines in the forward time direction approximately 121 logs of 10 from the Planck scale epoch to the present. Thus, in contrast to $\Lambda \mathrm{CDM}$ cosmology, there is no "cosmological constant problem" in the FSC model.

As speculated in the FSC book chapter summary, ongoing cosmological matter creation may be paired with a continual decline in the cosmological vacuum energy density, as a requirement for conservation of energy in such a finite isolated expanding system. It should be remembered that the details of matter creation in all cosmological models are a mystery. In FSC, matter creation is an ongoing process, whereas $\Lambda \mathrm{CDM}$ cosmologists generally assume that all matter was created nearly instantaneously. However, as a result, a major difference between the two models is that only $\Lambda C D M$ cosmology has a cosmological constant problem, based upon its embedded constant post-inflationary vacuum energy density assumption.

As a consequence of the dark energy observations, in addition to their cosmological constant and instantaneous matter creation assumptions, $\Lambda \mathrm{CDM}$ cosmologists must now also assume certain features of the universal expansion. These features had not been required when it was once thought (i.e., before 1998) that the cosmological vacuum energy density might actually be perpetually zero. They now require that universal expansion decelerated during the first half of the cosmic time span since the Big Bang, and then, almost imperceptibly, began to accelerate approximately 6 billion years ago. This becomes absolutely necessary if one requires a post-inflationary cosmological constant at the currently observed value of about $10^{-9} \mathrm{~J} \cdot \mathrm{m}^{-3}$. Nevertheless, this deceleration-followed-by-acceleration scenario of universal expansion is clearly debatable, especially when one considers the observational statistical error bars in Figure 2.

When one compares the relative luminosity and angular diameter distances between the two competing models, in the form of a ratio, it has recently been shown that the $\Lambda \mathrm{CDM}$ model contention of late cosmic acceleration could be an illusion produced by a $R_{h}=c t$ universe [17].

Further support that cosmic acceleration could be an illusion is clearly evident in Figure 1 of the current report. It is readily apparent that the FSC graph of the $\log$ of the Hubble parameter as a function of redshift $z$ is sinuous in appearance. We see the following: an upward flexion curve out to a $z$ value of about 1.0 (corresponding to the last 7.3 billion years of the FSC cosmic expansion); a roughly straight line segment for $1.0<z<1.7$ (corresponding to 3.76 to 7.3 billion years 
of cosmic age); and an opposite flexion curve for $z$ values greater than about 1.7 (corresponding to the first 3.76 billion years of the FSC cosmic expansion). The overall shape of the graph greatly resembles what $\Lambda \mathrm{CDM}$ proponents have been expecting for a scalar temporal behavior of dark energy. And yet, the FSC $R_{h}=c t$ model expansion, by definition, neither decelerates nor accelerates!

The upward curving portion of our Figure 1 graph out to a $z$ value of about 1.5 , is already largely filled in by the accumulated Type Ia supernovae data [18]. Not yet known are the exact Hubble parameter values at the cosmic times when these supernovae exploded. Fortunately, the coming decade of observational studies should give us a better idea of the precise scalar nature of the Hubble parameter.

Regardless, given the overall shape of our Figure 1 graph, it may well be that apparent early cosmic deceleration and late cosmic acceleration both ultimately prove to be illusions produced by a constant-velocity, linearly-expanding, FSC universe.

Given the ongoing tension between different research teams considering what current near and deep space observations might be telling us about the $H_{0}$ value as a snapshot in time, it is worth noting the following:

The 2018 Planck Collaboration analysis of the CMB looked at 99.998 percent of the current radius of the universe. Their consensus $H_{0}$ estimate of 67.36 $\mathrm{km} \cdot \mathrm{s}^{-1} \cdot \mathrm{Mpc}^{-1}$ appears, in FSC, to fit with a $14.6 \mathrm{Gyr}$ old universe. According to Table 1, the Freedman, et al. $H_{0}$ observation of $69.6 \mathrm{~km} \cdot \mathrm{s}^{-1} \cdot \mathrm{Mpc}^{-1}$ [19] appears to be fitted nicely to a $14 \mathrm{Gyr}$ estimated cosmic age. Whereas, the SHoES project $H_{0}$ observations of $74-77 \mathrm{~km} \cdot \mathrm{s}^{-1} \cdot \mathrm{Mpc}^{-1}$ [20] appear to be ideally fitted to a $13 \mathrm{Gyr}$ (or less) cosmic age. One need only consider the current $14.27+/-0.38 \mathrm{Gyr}$ best age estimate of the HD 140283 "Methuselah star" [21] to judge which current $H_{0}$ estimate is the most likely outlier.

\section{Summary and Conclusion}

Equations of FSC have been utilized to characterize the model's temporal behavior of dark energy. A table relating cosmic age, cosmological redshift, and the temporal FSC Hubble parameter value has been created. The resulting graph of the log of the Hubble parameter as a function of cosmological (or galactic) redshift has a particularly interesting sinuous shape: an upward flexion curve out to a $z$ value of about 1.0 (corresponding to the last 7.3 billion years of the FSC cosmic expansion); a roughly straight line segment for $1.0<z<1.7$ (corresponding to 3.76 to 7.3 billion years of cosmic age); and an opposite flexion curve for $z$ values greater than about 1.7 (corresponding to the first 3.76 billion years of the FSC cosmic expansion). The overall shape of the graph greatly resembles what $\Lambda \mathrm{CDM}$ proponents have been expecting for a scalar temporal behavior of dark energy. And yet, the FSC $R_{h}=c t$ model expansion, by definition, neither decelerates nor accelerates. It may well be that apparent early cosmic deceleration and late cosmic acceleration both ultimately prove to be illusions produced by a con- 
stant-velocity, linearly-expanding, FSC universe.

\section{Dedications and Acknowledgements}

This paper is dedicated to Dr. Stephen Hawking and Dr. Roger Penrose for their groundbreaking work on black holes and their possible application to cosmology. Dr. Tatum also thanks Dr. Rudolph Schild of the Harvard-Smithsonian Center for Astrophysics for his past support and encouragement. Author Seshavatharam UVS is indebted to professors Brahmashri M. NagaphaniSarma, Chairman, Shri K.V. Krishna Murthy, founding Chairman, Institute of Scientific Research in Vedas (I-SERVE), Hyderabad, India, and to Shri K.V.R.S. Murthy, former scientist IICT (CSIR), Govt. of India, Director, Research and Development, I-SERVE, for their valuable guidance and great support in developing this subject.

\section{Conflicts of Interest}

The authors declare no conflicts of interest regarding the publication of this paper.

\section{References}

[1] Aghanim, N., et al. (2018) Planck 2018 Results VI. Cosmological Parameters.

[2] Perlmutter, S., et al. (1999) Astrophysical Journal, 517, 565-586.

[3] Melia, F. (2012) Astronomical Journal, 144, 110. https://doi.org/10.1088/0004-6256/144/4/110

[4] Nielsen, J.T., et al. (2015) Scientific Reports, 6, Article No. 35596. https://doi.org/10.1038/srep35596

[5] Wei, J.-J., et al. (2015) Astronomical Journal, 149, 102. https://doi.org/10.1088/0004-6256/149/3/102

[6] Tutusaus, I., et al. (2017) Astronomy \& Astrophysics, 602, A73. https://doi.org/10.1051/0004-6361/201630289

[7] Dam, L.H., et al. (2017) Monthly Notices of the Royal Astronomical Society, 472, 835-851. https://doi.org/10.1093/mnras/stx1858

[8] Melia, F. (2019) Modern Physics Letters A, 33, Article No. 1930004. https://arxiv.org/abs/1904.11365 https://doi.org/10.1142/S0217732319300040

[9] Tatum, E.T. (2018) Journal of Modern Physics, 9, 1867-1882. https://doi.org/10.4236/jmp.2018.910118

[10] Tatum, E.T. (2019) A Heuristic Model of the Evolving Universe Inspired by Hawking and Penrose. In: Tatum, E., Ed., New Ideas Concerning Black Holes and the Universe, IntechOpen, London, 5-21. https://doi.org/10.5772/intechopen.75238

[11] Tatum, E.T. and Seshavatharam, U.V.S. (2015) International Journal of Astronomy and Astrophysics, 5, 133-140. https://doi.org/10.4236/ijaa.2015.53017

[12] Tatum, E.T. and Seshavatharam, U.V.S. (2018) Journal of Modern Physics, 9, 1404-1414. https://doi.org/10.4236/jmp.2018.97085

[13] Tatum, E.T. (2019) Journal of Modern Physics, 10, 974-979. https://doi.org/10.4236/jmp.2019.108064 
[14] Tatum, E.T. and Seshavatharam, U.V.S. (2018) Journal of Modern Physics, 9, 2008-2020. https://doi.org/10.4236/jmp.2018.910126

[15] Perlmutter, S. (2016) Supernova Cosmology Project. Lawrence Berkeley National Laboratory, Berkeley. http://www.supernova.lbl.gov

[16] Tatum, E.T. and Seshavatharam, U.V.S. (2018) Journal of Modern Physics, 9, 1469-1483. https://doi.org/10.4236/jmp.2018.98091

[17] Tatum, E.T. and Seshavatharam, U.V.S. (2018) Journal of Modern Physics, 9, 1397-1403. https://doi.org/10.4236/jmp.2018.97084

[18] Betoule, M., et al. (2014) $A \& A, 568$, A22.

[19] Freedman, W.L., et al. (2020) Astrophysical Journal, 891, 57-75. https://doi.org/10.3847/1538-4357/ab7339

[20] Reiss, A.G., et al. (2016) A 2.4\% Determination of the Local Value of the Hubble Constant.

[21] Vandenberg, D.A., et al. (2014) Astrophysical Journal, 792, Art. No. 110. https://doi.org/10.1088/0004-637X/792/2/110 